\title{
Diphoton production in vector-boson scattering at the LHC at next-to-leading order QCD
}

\author{
Francisco Campanario, ${ }^{a}$ Matthias Kerner, ${ }^{b}$ Le Duc Ninh ${ }^{c}$ and Ivan Rosario ${ }^{a}$ \\ ${ }^{a}$ Theory Division, IFIC, University of Valencia-CSIC, \\ E-46980 Paterna, Valencia, Spain \\ ${ }^{b}$ Physik-Institut, Universität Zürich, \\ Winterthurerstrasse 190, 8057 Zürich, Switzerland \\ ${ }^{c}$ Institute For Interdisciplinary Research in Science and Education, \\ ICISE, 590000 Quy Nhon, Vietnam \\ E-mail: francisco.campanario@ific.uv.es, mkerner@physik.uzh.ch, \\ ldninh@ifirse.icise.vn, ivan.rosario@ific.uv.es
}

ABSTRACT: In this paper, we present results at next-to-leading order (NLO) QCD for photon pair production in association with two jets via vector boson scattering within the Standard Model (SM), and also in an effective field theory framework with anomalous gauge coupling effects via bosonic dimension- 6 and 8 operators. We observe that, compared to other processes in the class of two electroweak (EW) vector boson production in association with two jets, more exclusive cuts are needed in order to suppress the SM QCD-induced background channel. As expected, the NLO QCD corrections reduce the scale uncertainties considerably. Using a well-motivated dynamical scale choice, we find moderate $K$-factors for the EW-induced process while the QCD-induced channel receives much larger corrections. Furthermore, we observe that applying a cut of $\Delta \phi_{j_{2} \gamma_{1}}^{\text {cut }}<2.5$ for the second hardest jet and the hardest photon helps to increase the signal significance and reduces the impact of higher-order QCD corrections.

KEYwORDS: NLO Computations

ARXiv EPrint: 2002.12109 


\section{Contents}

1 Introduction 1

2 Calculational setup $\quad 3$

3 Phenomenological results 5

$\begin{array}{llr}4 & \text { Conclusions } & 19\end{array}$

\section{Introduction}

Vector boson pair production in association with two jets, denoted as $V V j j$ in the following, has been studied in detail in recent years, both from the theoretical and the experimental sides of the Large Hadron Collider (LHC) physics community, since it provides information on weak boson scattering and because it is sensitive to beyond Standard Model (SM) physics via anomalous gauge boson couplings.

From the theoretical point of view, $\gamma \gamma j j$ events can be produced at leading order (LO) via electroweak (EW)-induced channel of order $\mathcal{O}\left(\alpha^{4}\right)$, QCD-induced channel of order $\mathcal{O}\left(\alpha_{s}^{2} \alpha^{2}\right)$, and the interference between them of order $\mathcal{O}\left(\alpha_{s} \alpha^{3}\right)$. The EW-induced channel is considered here to be the signal since it is sensitive to the weak boson scattering and to EW quartic gauge couplings, figure 1. The QCD-induced channel is an irreducible background. Despite the apparent $\mathcal{O}\left(\alpha^{2} / \alpha_{s}^{2}\right)$ suppression factor of the EW mechanism, once appropriate kinematical cuts are applied, both mechanisms yield integrated cross sections of the same order, and they provide distinct differential distributions in selected observables. Therefore, the EW mechanism turns out to be an excellent channel to test the SM and search for hints of beyond SM physics.

Using the vector boson scattering (VBS) cuts defined in section 3 , the relative contributions at LO of the EW, QCD and interference contributions are $46 \%, 53 \%$, and $1 \%$, respectively (see table 1). At next-to-leading order (NLO) QCD, the distinction between EW- and QCD-induced channels becomes more blurry as new interference terms of the order $\mathcal{O}\left(\alpha_{s}^{2} \alpha^{3}\right)$ occur, mixing the two mechanisms together. Nevertheless, it can be safely assumed that all interference effects between the EW- and QCD-induced mechanisms are still negligible at NLO QCD, given the fact the NLO QCD scale uncertainties are at the level of $20 \%$ on the QCD-induced cross section, see table 4.

The EW-induced mechanism can be further classified into $s$-channel contributions, which can be considered as a triple EW boson production with a subsequent hadronic decay of a vector boson, i.e. $\gamma \gamma V \rightarrow \gamma \gamma j j$, and the $t / u$-channel VBS. In the VBS approximation defined in ref. [1], which we will use to calculate the VBS signal at NLO QCD, the $s$ channel and its interference with the $t / u$-channels will be neglected because these effects 
are very small compared to the scale uncertainties of the QCD background when VBS cuts are applied. To further reduce the impact of the $s$-channel contributions, we will remove a window of $15 \mathrm{GeV}$ around the $V \rightarrow$ jets $\left(M_{V}=\left(M_{W}+M_{Z}\right) / 2\right)$ resonances. Moreover, since the interference between the $t$ and $u$ channels, occurring for sub-processes with identical quark lines, is negligible, the VBS approximation includes the contributions from the $t$ and $u$ channels independently.

Measuring the VBS signal is now an active field of research using Run-2 data at the LHC. Recently, $13 \mathrm{TeV}$ results from ATLAS and CMS measuring the EW-induced channels involving two massive vector bosons with leptonic decays have been published for the same-sign $W W j j[2,3], W Z j j[4,5]$, and $Z Z j j$ [6], showing agreement with the SM predictions. In this context, we would like to mention that NLO QCD corrections to the massive EW channels have been known for a decade in the VBS approximation [711], and have also been recently more precisely calculated beyond the VBS approximation with full off-shell and interference effects taken into account in ref. [12] (see also ref. [13]) for the same-sign $W W j j$ process and in ref. [14] for the $W Z j j$ channel. The $s$-channel contributions at an approximate NLO QCD accuracy [15] are available in the VBFNLO package [16-18], a flexible parton-level Monte Carlo program which allows to define general acceptance cuts and kinematic distributions. They were first computed in the framework of triple vector boson production with subsequent leptonic decays in refs. [19-22]. The NLO QCD predictions for the massive QCD-induced mechanisms are also available in refs. [12, 23-28]. Moreover, NLO EW corrections have been calculated for the same-sign $W W j j$ process $[12,29,30]$ (including $\mathrm{EW}, \mathrm{QCD}$ and all interference) and EW $W Z j j$ channel [14].

The EW induced $Z_{\gamma j j}[31-33]$ and $W^{ \pm} \gamma j j$ [34] channels have also been measured by the ATLAS and CMS experiments. Again, no deviation with the SM predictions was found. The NLO QCD corrections to the EW processes were calculated in refs. $[35,36]$ in the VBS approximation. For the QCD channels, the NLO QCD corrections were computed in refs. $[37,38]$. The $s$-channel NLO QCD predictions are also available in the VBFNLO package. They were first computed in refs. $[21,22]$ for the leptonic decay modes and in ref. [15] the hadronic decays were included.

EW di-photon production in association with two jets, $\gamma \gamma j j$, is the only process for which the NLO QCD predictions have not been studied and measurements are not available. It is an important process providing additional information on the EW boson scatterings and to beyond standard model physics via anomalous gauge boson couplings. Results at NLO QCD for the QCD-induced process have already been calculated in refs. [39-41].

In this paper, we present results at NLO QCD for the VBS $t / u$-channel for the process

$$
p p \rightarrow \gamma \gamma j j+X, \quad " \gamma \gamma j j "
$$

in the VBS approximation. Representative diagrams are shown in figure 1. In the upper left diagram, the sensitivity of the process to vector boson scattering and to weak quartic gauge boson couplings is manifest. Using an effective field theory (EFT) approach, we have also included at NLO QCD the effect of dimension- 6 and 8 operators involving the EW 

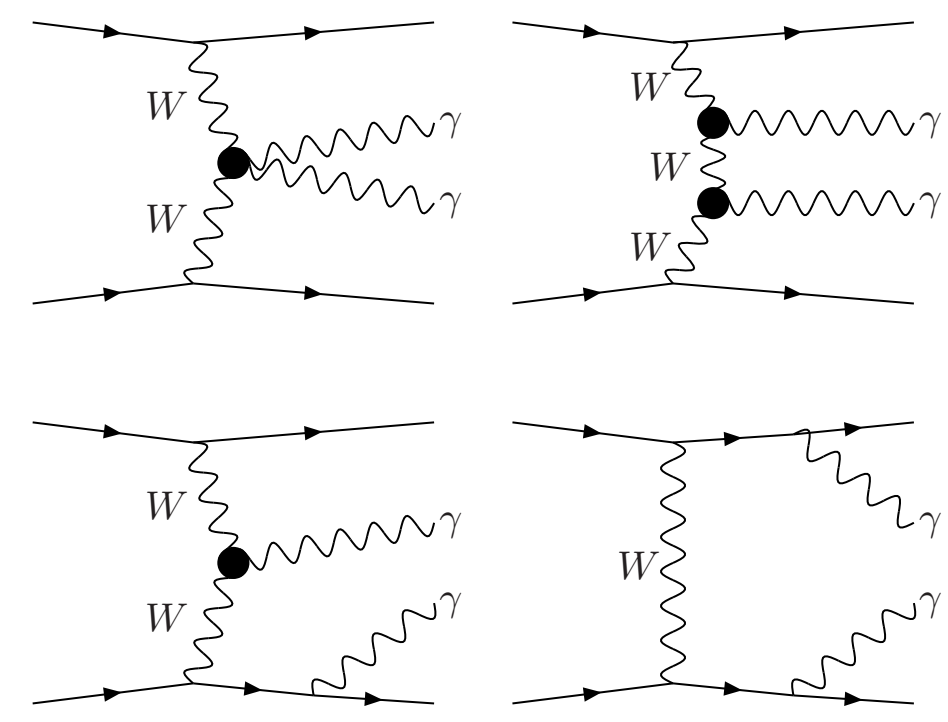

Figure 1. Representative Feynman diagrams at LO. With dark dots, we highlight the sensitivity to electroweak triple and quartic gauge couplings of the process.

gauge bosons and the Higgs field. While dimension-6 operators also contribute here they are better investigated in vector-boson-pair production with much higher statistics.

Besides, we have also computed the SM NLO QCD-induced channel, which is used here as the background process. Both EW- and QCD-induced channels have been implemented in the VBFNLO package, which will be available in the new release of the program or upon request. In addition, for a set of selected processes such as the complete list of EW $V V j j$ processes, it can be linked at NLO to the Herwig [42] event generator to study shower and hadronization effects.

The paper is organized as follows: section 2 describes the method used to compute the cross sections. Section 3 presents phenomenological results at the integrated cross section level and for differential distributions. Furthermore, as an illustrative example, the differential distribution of the diphoton invariant mass including anomalous couplings effects from an dimension- 8 operator is shown. Finally, conclusions are presented in section 4 .

\section{Calculational setup}

In this section, we shortly describe the method used to calculate $\gamma \gamma j j$ production, both for the EW VBS channel and the QCD mechanism. We closely follow the strategy of other similar EW and QCD processes implemented in VBFNLO. The codes are based on a simplification of the VBS and QCD $\ell^{+} \ell^{-} \gamma j j$ processes (called EW and QCD $Z_{\ell} \gamma j j$ for simplicity from now on).

We work in the five-flavor scheme and top-quark loops are taken into account in the QCD mechanism. We note that sub-processes with external bottom quarks are included as long as there is no external top quark involved. This means that for the EW-induced 
channel, the bottom quark is included in the neutral currents but not in the charged currents. For the propagator of the massive vector bosons, a fixed width is used for both resonant and non-resonant propagators. The weak-mixing angle and other coupling constants are kept at real values, i.e. complex masses are not used to calculate the weak-mixing angle. Photon fragmentation functions are not included and we use instead the Frixione smooth-cone isolation criteria [43] for the external photons.

For the QCD-induced channel, the gauge invariant set of diagrams with one or two photon directly attached to a closed-quark loop is discarded. We have estimated this contribution to be about $-0.3 \%$ of the NLO cross section. Closed-quarks loops with two or three gluons are included.

For the EW process, we work in the VBS approximation and consider only the $t / u$ channels Feynman diagrams. Interference effects between the $t$ - and $u$-channel diagrams as well as with the $s$-channel contributions are neglected. A detailed study on the validity of this approximation for the same-sign $W W j j$ production channel can be found in ref. [13] - the accuracy of the VBS approximation once tight VBS cuts are applied should be enough for present and near future experiments, being around the few-percent level. Consistently, virtual corrections with a gluon exchange between the two quark lines are not considered since, due to the color structure of the amplitudes, they are only non-vanishing for the interference of the $t$ - and $u$-channel diagrams, which are phase-space suppressed and neglected in the VBS approximation.

In the following, we give a brief description of the technical details implemented to generate the code for the EW process - the QCD induced channel has been obtained using a similar procedure. As mentioned above, our code is based on a simplification of the VBS $\ell^{+} \ell^{-} \gamma j j$ amplitudes already programmed in VBFNLO. We therefore first discuss the implementation of this process. We use the effective current approach and the spinhelicity formalism of refs. [44, 45], which allows to factorize the EW-dependent leptonic tensors from the QCD amplitudes. The lepton pair can either originate from the decay of an intermediate boson, $V_{1}=Z / \gamma^{*} \rightarrow \ell^{+} \ell^{-}$or $\hat{V}=Z / \gamma^{*} \rightarrow \ell^{+} \ell^{-} \gamma$, which is radiated off the quark lines, or it can stem from the scattering of the $t$-channel vector bosons, e.g. $V V / W^{+} W^{-} \rightarrow \ell^{+} \ell^{-}$. We denote the latter as leptonic tensor $T_{\ell \ell}$ and we define the tensors $T_{\gamma}$ and $T_{\ell \ell \gamma}$ in a similar way. Using this notation, the $\ell^{+} \ell^{-} \gamma j j$ amplitude can be written as the sum of the generic $q q \rightarrow V_{1} \gamma q q, q q \rightarrow \hat{V} q q, q q \rightarrow V_{1} T_{\gamma} q q, q q \rightarrow T_{\ell \ell} \gamma q q$, and $q q \rightarrow T_{\ell \ell \gamma} q q$ contributions. All spin correlation and off-shell effects are taken into account in the definition of the leptonic tensors and decay currents.

With this approach, it is trivial to obtain the $\gamma \gamma j j$ code. We simply select the same generic amplitudes then replace $V_{1}=\gamma_{2}$ and $\hat{V}=0$ in the effective currents. In the amplitudes containing the leptonic tensors, we set $T_{\ell \ell}=T_{\gamma_{2}}, T_{\ell \ell \gamma}=0$. We use the subindex 2 to distinguish the final state photons. Finally, we define new leptonic tensors $V V / W^{+} W^{-} \rightarrow \gamma \gamma \equiv T_{\gamma \gamma}$, and use it in the $q q \rightarrow T_{\gamma \gamma} q q$ amplitude.

We have performed several tests to validate our code. The LO and real radiation matrix elements have been cross-checked with Madgraph [46] at the amplitude level and with Sherpa $[47,48]$ for integrated cross sections, finding agreement at the machine precision and per mille level, respectively. For the EW-induced process, we have subtracted the 
$s$-channel contributions from the real matrix elements in Sherpa, otherwise, percent-level agreement is found for typical VBS cuts. The impact of the neglected $s$-channel is only noticeable in the real corrections, thus, its effect at the total NLO QCD cross section should be below the percent level. Additionally, for the virtual contributions, the factorization of the poles, gauge invariance and independence from the dimensional-regularization scale [45] have been proved at the machine precision level for some building blocks. For the realemission part, the convergence of the Catani-Seymour subtraction algorithm has also been checked. Finally, the numerical stability of the code is controlled via Ward identities. For the EW process the amplitude is set to zero, if the identities are not satisfied at the per mille level using double precision. For the QCD process, we have a rescue system in quadruple precision, and the amplitude is only set to zero for the points that do not satisfy the Ward identities at the per mille level in quadruple precision. The fraction of points for which this takes place is at the per mille level in the EW process. Since the contribution of the virtual corrections, after the cancellation of the infrared divergences, is about a few percent, the error induced by this procedure is irrelevant. For the QCD channel, the fraction of rejected points is well below the per mille level, thus, completely negligible.

Additionally, for the QCD-induced mechanism, using the setup described in ref. [40], we obtain $\left[\sigma_{\mathrm{LO}}=2.045(1), \sigma_{\mathrm{NLO}}=2.714(3)\right] \mathrm{pb}$, to be compared with $[2.046(2), 2.691(7)] \mathrm{pb}$ of ref. [40], which includes the fermion loops with one or two photons coupling to it. For the sake of comparison, we have estimated this fermion-loop contribution separately and obtained $\sigma_{\text {fer. loop }}^{\gamma, \gamma \gamma} \approx-7.8 \mathrm{fb}$, giving an agreement at the 2 standard-deviation level for the NLO cross section. Furthermore, the top-quark loop contribution in the gluon self-energies and three-gluon vertices is included in our calculation while being omitted in ref. [40]. This small effect may contribute to the above small discrepancy.

Concerning the anomalous-gauge-coupling implementation, new physics effects only occur in the photonic tensors $T_{\gamma}, T_{\gamma_{2}}$, and $T_{\gamma \gamma}$. For dimension- 6 and 8 operators, which have also been implemented for the other EW $V V j j$ processes in the VBFNLO program [18, 49, 50], we have crosschecked our implementation at the LO-amplitude level against Madgraph with the FeynRules [51, 52] model file EWdim6 [50, 53, 54] (for dimension-6) and with the FeynRules model files for quartic-gauge couplings [55, 56] (for dimension-8). Agreement at the machine-precision level has been found at random phase-space points.

\section{Phenomenological results}

We use the following SM input parameters [57]

$$
\begin{aligned}
G_{F} & =1.1663787 \times 10^{-5} \mathrm{GeV}^{-2}, & M_{W} & =80.379 \mathrm{GeV}, \\
M_{Z} & =91.1876 \mathrm{GeV}, & M_{t} & =172.9 \mathrm{GeV},
\end{aligned}
$$

from which the electromagnetic coupling is calculated as $\alpha=\sqrt{2} G_{F} M_{W}^{2}\left(1-M_{W}^{2} / M_{Z}^{2}\right) / \pi$ and the widths as $\Gamma_{Z}=2.507426 \mathrm{GeV}, \Gamma_{W}=2.096211 \mathrm{GeV}$. The mass of all the other light fermions are neglected as the results are insensitive to them. The top-quark mass dependence occurs via the fermion-loop corrections in the QCD-induced channels. This 
contribution is known to be very small, hence the results depend very weakly on the topquark mass. The Cabibbo-Kobayashi-Maskawa matrix is set to unity in our calculations.

Concerning kinematic cuts, we require

$$
\begin{aligned}
p_{T, j} & >30 \mathrm{GeV}, & \left|y_{j}\right| & <4.5, \\
p_{T, \gamma} & >30 \mathrm{GeV}, & \left|y_{\gamma}\right| & <2.5, \\
\Delta R_{\gamma \gamma} & >0.4, & \Delta R_{j \gamma} & >0.8,
\end{aligned}
$$

where jets are reconstructed from massless partons satisfying $\left|y_{\text {parton }}\right|<5$ using the anti- $k_{t}$ algorithm [58] with the radius parameter $R=0.4, y$ denoting the rapidity. In this paper we define the $R$-separation between two particles $a$ and $b$ as $\Delta R_{a b}=\sqrt{\left(\Delta y_{a b}\right)^{2}+\left(\Delta \phi_{a b}\right)^{2}}$ where $\Delta y_{a b}=\left|y_{a}-y_{b}\right|$ (here and the following) and $\Delta \phi_{a b}=\left|\phi_{a}-\phi_{b}\right| \leq \pi$. As default in VBFNLO, to remove the photon singularity at $q^{2}=0$ where $q$ is the momentum of a $t$ channel gauge-boson exchange between the two quark lines, a technical cut of $q^{2}>4 \mathrm{GeV}^{2}$ is applied for the EW process where the singularity occurs. This is expected to be a very good approximation.

In order to isolate prompt photon events and minimize the parton-to-photon fragmentation contribution, we use Frixione's smooth-cone isolation criteria [43]. Events are accepted if

$$
\sum_{i \in \text { partons }} p_{T, i} \theta\left(R_{\gamma j}-R_{\gamma i}\right) \leq \epsilon p_{T, \gamma} \frac{1-\cos R_{\gamma j}}{1-\cos \delta_{0}} \forall R_{\gamma j}<\delta_{0},
$$

where the index $j$ runs over all partons, $\delta_{0}=0.4$ is the cone-radius parameter, and $\epsilon$ is the efficiency. The notation $R_{\gamma j}=\Delta R_{\gamma j}$ has been used for shortness. We choose as default $\epsilon=0.05$, following the recommendation of tight-isolation cuts in ref. [59]. Note that eq. (3.3) must be applied independently for the two photons. We see clearly from eq. (3.3) that soft gluons are accepted while a hard quark exactly collinear to a photon is rejected, thereby ensuring IR safety while removing the collinear contribution. The cut $\Delta R_{j \gamma}>0.8$ in eq. (3.2) helps to isolate the photons further from the jets. In our calculation, since the quark-photon collinear events have been discarded, no fragmentation contribution occurs. In experiments, the above smooth-cone isolation criteria cannot be exactly implemented due to the finite resolution of the detector. However, using a tight-isolation cut both in theoretical calculations and measurements (with either a standard-cone cut or a discretized version of the smooth-cone criteria) is expected to produce a very good agreement at the few-percent level, according to the study in ref. [59] for $p p \rightarrow \gamma \gamma$. This is because the tight cut suppresses the fragmentation contribution, where a photon usually lies inside a hadronic jet.

To enhance the signal, we employ further the following VBS cuts

$$
m_{j_{1} j_{2}}>800 \mathrm{GeV},\left|y_{j_{1}}-y_{j_{2}}\right|>3, \quad y_{j_{1}} y_{j_{2}}<0,
$$

where $j_{1}$ and $j_{2}$ are the two tagging jets ordered by $p_{T}$ with $j_{1}$ being the hardest jet. Furthermore, the photons are required to fall inside the rapidity gap of the two tagging jets, i.e. $y_{\min }<y_{\gamma_{i}}<y_{\max }$ with $y_{\min }=\min \left(y_{j_{1}}, y_{j_{2}}\right), y_{\max }=\max \left(y_{j_{1}}, y_{j_{2}}\right)$. Additionally, 


\begin{tabular}{|c|c|c|c|c|c|}
\hline & VBS & full EW & QCD & Interf. & All \\
\hline$\sigma_{\mathrm{LO}}[\mathrm{fb}]$ & $24.929(6)$ & $24.94(8)$ & $21.664(10)$ & $0.542(2)$ & $47.15(8)$ \\
\hline$\Delta[\%]$ & 52.8 & 52.9 & 46.0 & 1.1 & 100 \\
\hline
\end{tabular}

Table 1. VBS, full EW and QCD cross sections and the EW-QCD interference at LO. The relative contributions are also shown. The full EW and interference cross sections are obtained using Sherpa, while the VBS and the QCD results are from our VBFNLO program.

to remove the $p p \rightarrow V \gamma \gamma(V \rightarrow j j)$ contribution with $V=W, Z$, we accept only events satisfying

$$
\left|m_{\text {jets }}-\frac{M_{W}+M_{Z}}{2}\right|>15 \mathrm{GeV}
$$

where $m_{\text {jets }}$ can be the mass of any massive jet or the invariant mass of any combination of two or more jets. Note that we have $m_{3 \text { jets }}>800 \mathrm{GeV}$ due to the invariant mass cut in eq. (3.4) in the default setup. However, when the value of the $m_{j_{1} j_{2}}$ cut becomes smaller than $\left(M_{W}+M_{Z}\right) / 2+15 \mathrm{GeV}$ as can happen in the scan shown in figures 4 and 10 , then the three-jet contribution is affected by the cut in eq. (3.5). The $\Delta y_{j_{1} j_{2}}$ cut choice together with this last cut should guarantee the validity of the VBS approximation at the percent level, as demonstrated in EW-Hjjj production [60], independently of the di-jet invariant mass cut used.

To calculate hadronic cross sections, parton distribution functions (PDF) and the strong coupling constant $\alpha_{s}\left(\mu_{R}\right)$ are calculated using the LHAPDF6 program [61] with the PDF4LHC15_nlo_100 set [62-65]. The same PDF set is used both for the LO and NLO results. Our default choice for the renormalization and factorization scales is $\mu_{R}=\mu_{F}=$ $H_{T} / 2$ with

$$
H_{T}=\sum_{i \in \text { partons }} p_{T, i}+p_{T, \gamma_{1}}+p_{T, \gamma_{2}}
$$

for both EW and QCD-induced contributions (see the scale-dependence discussion in the next section).

In the following, SM results for the LHC at $\sqrt{s}=13 \mathrm{TeV}$ will be first presented. After that we will show briefly results with anomalous gauge couplings from dimension- 8 operators as an illustration of the capabilities of our computer program.

With the above setup, we first show in table 1 the full LO cross section with full EW amplitudes ( $t, u, s$ channels included), QCD amplitudes and their interference. We see that the EW-QCD interference is $1.1 \%$, completely negligible compared to the scale uncertainties of the QCD cross section discussed below. In addition, the VBS approximation is also provided, showing an excellent agreement with the full EW result.

From now on we will use the notation EW to denote the VBS approximation. Within the default VBS cuts, we expect that the difference between the EW and the full EW results is completely invisible also at $\mathrm{NLO}$ QCD, given the fact that the QCD corrections are very small. 


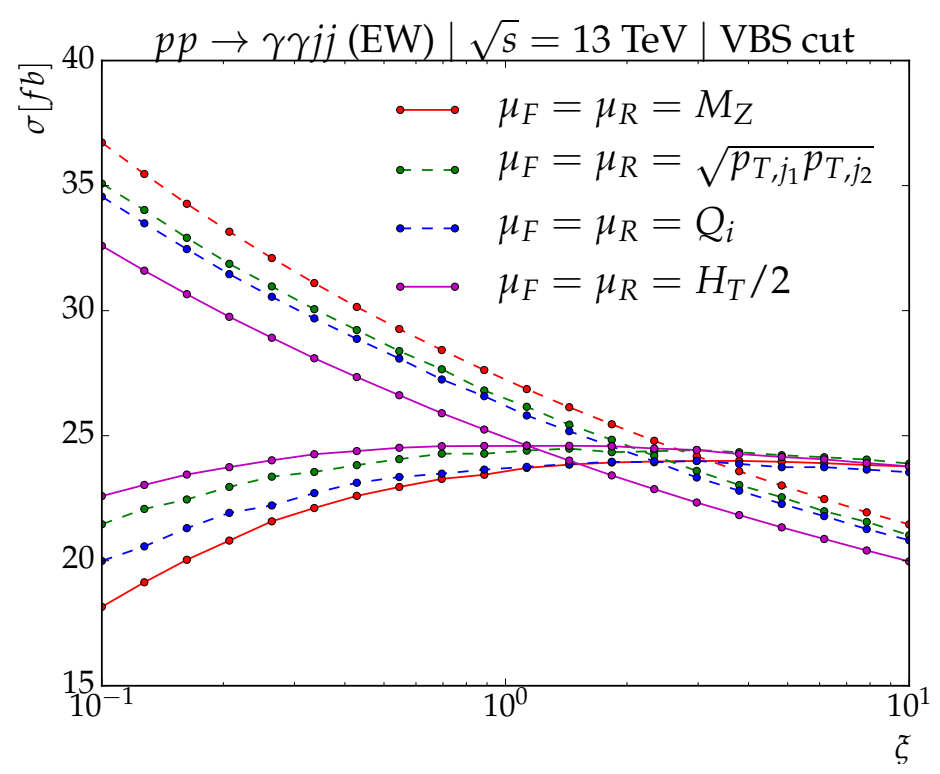

Figure 2. Scale dependence of the LO and NLO cross sections for the EW-induced channel.

We now discuss the scale dependence of the integrated cross sections. Setting $\mu_{F}=$ $\mu_{R}=\xi \mu_{0}$, the scale dependence is shown in figure 2 for the EW-induced process. The scale dependence of the QCD-induced process has already been provided in refs. [39-41], showing that $H_{T} / 2$ is a good scale choice. For the sake of comparisons, results with a fixed scale choice centered around $\mu_{0}^{\mathrm{fix}}=M_{Z}$ and with other dynamical scale choices $\sqrt{p_{T, j_{1}} p_{T, j_{2}}}$ and $Q_{i}$ are also shown for the EW case. $Q_{i}$ is the momentum transfer from quark line 'i' and is set independently for both quark lines. At LO, there is no $\mu_{R}$, hence the dependence comes from $\mu_{F}$ via the PDFs. The scale choice $H_{T} / 2$ gives the smallest correction around the central scale $\mu_{0}$ when moving from LO to NLO and, in this view, the scale $\sqrt{p_{T, j_{1}} p_{T, j_{2}}}$ comes second, being more consistent than the $Q_{i}$ scale. Moreover, the dependence on $\xi$ at NLO shows that $H_{T} / 2$ and $\sqrt{p_{T, j_{1}} p_{T, j_{2}}}$ provide the most stable behavior. From this evidence, we conclude that $H_{T} / 2$ is a good scale choice for calculating the EW cross sections in the present setup. The scale dependence for EW $p p \rightarrow Z \gamma j j$ presented in ref. [36], for a similar setup, also shows that $H_{T} / 2$ gives the most stable behavior at NLO.

We next study the dependence of the cross sections on the photon-isolation parameters. Results are shown in figure 3 and table 2 . In figure 3 , we show the dependence of the EW and QCD cross sections on the $\epsilon$ parameter defined in eq. (3.3) for two cases $\delta_{0}=0.4$ and 0.7. Note that, for all cases the LO cross section is independent of $\delta_{0}$ and of $\epsilon$ because of the $\Delta R_{j \gamma}>0.8$ cut. At NLO, one additional partonic radiation occurs. This radiation is included or excluded depending on the values of $\delta_{0}$ and $\epsilon$. Only events with at least a parton in the vicinity of a photon satisfying $\Delta R_{\gamma, \text { parton }}<\delta_{0}$ can be rejected. This explains why the NLO cross section decreases as $\delta_{0}$ increases. It also explains why the cross section is more sensitive to $\epsilon$ when the cone-radius $\delta_{0}$ is larger. Numerically, we find that the EW cross section increases about $2 \%(6 \%)$ when varying $\epsilon \in(0.01,1)$ for $\delta_{0}=0.4(0.7)$. For 


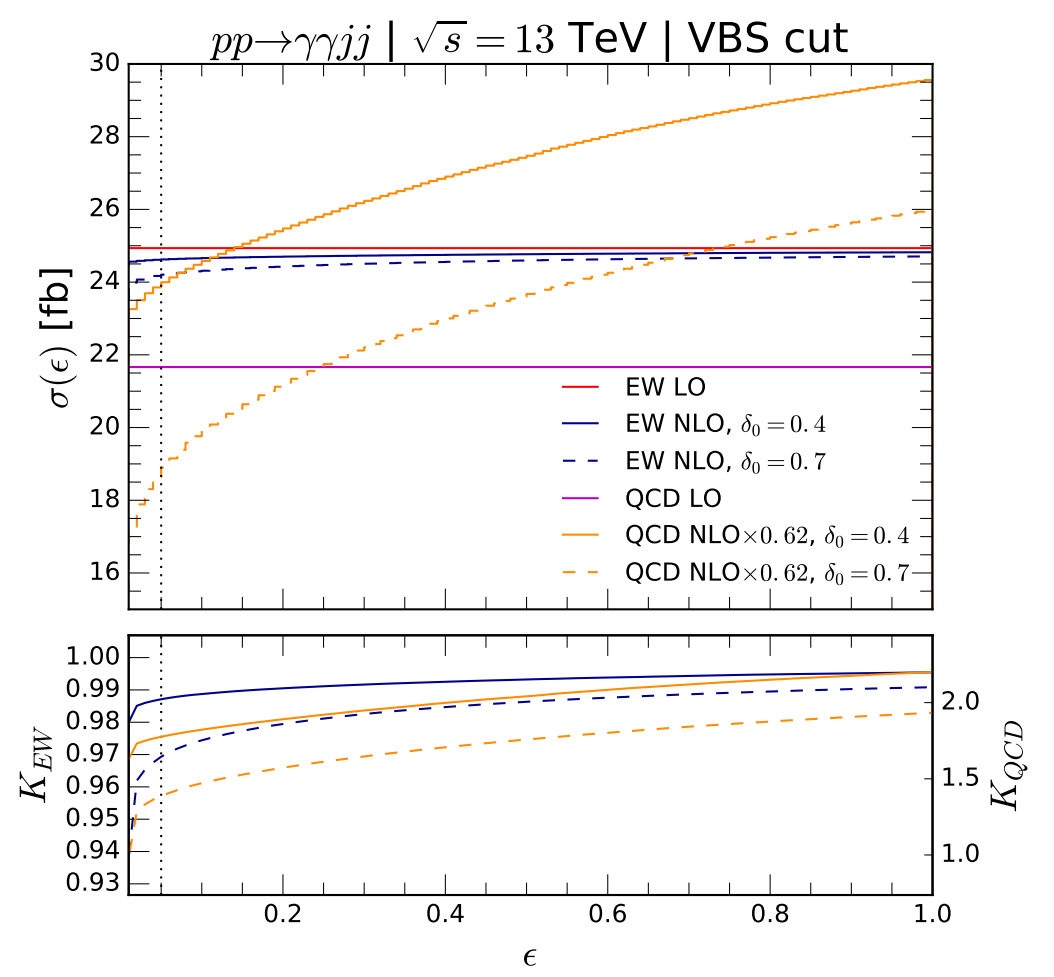

Figure 3. Dependence on the photon-isolation parameter $\epsilon$ defined in eq. (3.3) of the EW and QCD cross sections.

\begin{tabular}{|c|c|c|c|c|c|}
\hline$\delta_{0}$ & $\epsilon$ & EW NLO $[\mathrm{fb}]$ & $K_{\mathrm{EW}}$ & QCD NLO $[\mathrm{fb}]$ & $K_{\mathrm{QCD}}$ \\
\hline \multirow{5}{*}{0.4} & 0.01 & 24.4 & 0.98 & 35 & 1.63 \\
& 0.05 & 24.6 & 0.99 & 39 & 1.78 \\
& 0.5 & 24.8 & 0.99 & 44 & 2.04 \\
& 1.0 & $24.82(1)$ & 1.00 & $47.7(1)$ & 2.20 \\
\hline \multirow{5}{*}{0.7} & 0.01 & 23.4 & 0.94 & 21 & 0.98 \\
& 0.05 & 24.2 & 0.97 & 30 & 1.39 \\
& 0.5 & 24.6 & 0.99 & 38 & 1.76 \\
& 1.0 & $24.705(9)$ & 0.99 & $41.9(1)$ & 1.93 \\
\hline
\end{tabular}

Table 2. EW and QCD $p p \rightarrow \gamma \gamma j j$ cross sections at different values of photon-isolation parameters. The numbers in the parentheses are the statistical errors. EW and QCD LO cross sections, being independent of those parameters, are 24.929(6) fb and 21.664(10) fb, respectively.

the QCD channel, we have 35\% (97\%), correspondingly. We observe that the dependence on $\epsilon$ is significantly milder for the smaller cone radius. However, the $K$-factors defined as $\sigma_{N L O} / \sigma_{L O}$ are larger, in particular for the QCD channel, when the cone radius is decreased.

In experimental analyses, it is important to find out an optimal set of cuts to enhance the EW-induced channel. For this purpose, we show in figure 4 and table 3 the dependence of the significance defined as $S=\mathrm{EW} / \sqrt{\mathrm{QCD}}$, where $\mathrm{EW}$ and QCD represent the number of events of the two production mechanisms, calculated at NLO with luminosity $L=1 \mathrm{fb}^{-1}$, 


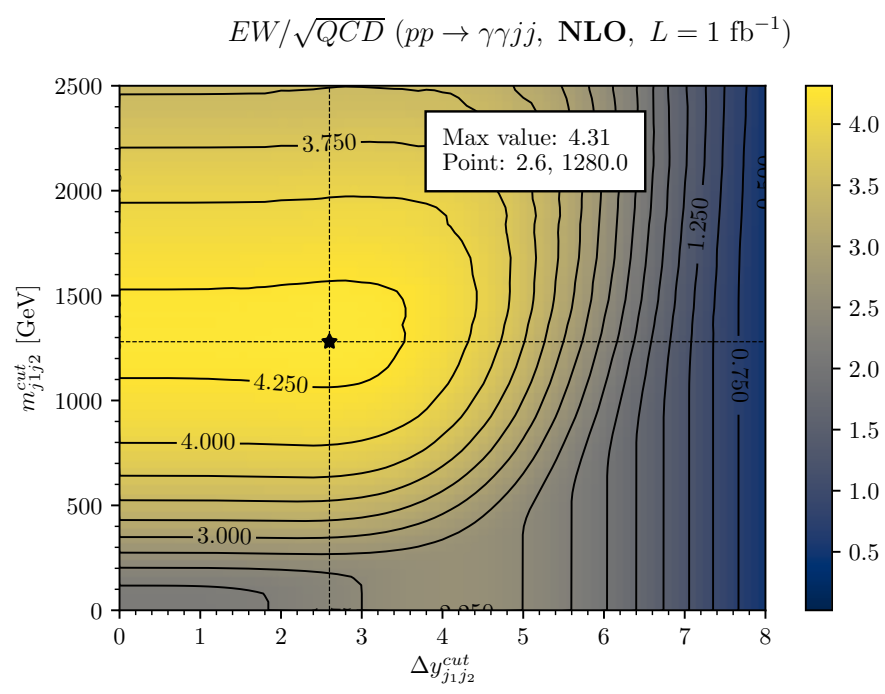

Figure 4. Dependence of the NLO significance EW $/ \sqrt{\mathrm{QCD}}$ calculated with luminosity $L=1 \mathrm{fb}^{-1}$ on the cuts $m_{j_{1} j_{2}}>m_{j_{1} j_{2}}^{\text {cut }}$ and $\Delta y_{j_{1} j_{2}}>\Delta y_{j_{1} j_{2}}^{\text {cut }}$. For the other cuts, default values are used.

\begin{tabular}{|c|c|c|c|}
\hline$m_{j_{1} j_{2}}^{\text {cut }}[\mathrm{GeV}], \Delta y_{j_{1} j_{2}}^{\text {cut }}$ & EW & QCD & $\mathrm{EW} / \sqrt{\mathrm{QCD}}$ \\
\hline 800,2 & $25.41(2)$ & $40.68(10)$ & 3.98 \\
\hline 800,3 & $24.62(1)$ & $38.53(3)$ & 3.97 \\
\hline 800,4 & $21.29(1)$ & $33.23(8)$ & 3.69 \\
\hline 600,3 & $30.69(2)$ & $72.8(2)$ & 3.60 \\
\hline 1000,3 & $19.36(2)$ & $21.66(6)$ & 4.16 \\
\hline
\end{tabular}

Table 3. EW and QCD event numbers for different cuts calculated at NLO with luminosity $L=1 \mathrm{fb}^{-1}$. For the other cuts, default values are used.

\begin{tabular}{|c|c|c|c|}
\hline Process & EW & QCD & EW $/ \sqrt{\mathrm{QCD}}$ \\
\hline$\gamma \gamma j j$ & $24.62(1)_{-0.59 \%}^{+0}$ & $38.53(3)_{-16 \%}^{+19 \%}$ & 4.0 \\
\hline$\ell^{+} \ell^{-} \gamma j j$ & $1.786(1)_{-0.84 \%}^{+0}$ & $0.883(2)_{-10 \%}^{+10 \%}$ & 1.9 \\
\hline$\ell^{+} \nu_{\ell} \gamma j j$ & $9.009(7)_{-0.79 \%}^{+0}$ & $8.87(3)_{-33 \%}^{+10 \%}$ & 3.0 \\
\hline$\ell^{-} \bar{\nu}_{\ell} \gamma j j$ & $5.401(4)_{-0.61 \%}^{+0}$ & $6.53(2)_{-24 \%}^{+6 \%}$ & 2.1 \\
\hline
\end{tabular}

Table 4. EW and QCD event numbers for different processes calculated at NLO with luminosity $L=1 \mathrm{fb}^{-1}$. For the charged lepton final states, both electron and muon are taken into account $(\ell=e, \mu)$ and the cross sections are calculated using VBFNLO version 3.0.0 beta 4. The scale uncertainties in percentage are also provided.

using the cuts $m_{j_{1} j_{2}}>m_{j_{1} j_{2}}^{\text {cut }}$ and $\Delta y_{j_{1} j_{2}}>\Delta y_{j_{1} j_{2}}^{\text {cut }}$. For the other cuts, default values are used. We see that the maximal significance region of $S>4.25$ is $m_{j_{1} j_{2}}^{\text {cut }} \in(1.1,1.6) \mathrm{TeV}$ and $\Delta y_{j_{1} j_{2}}^{\text {cut }}<3.5$. If we require $S>3.75$ then the region becomes $m_{j_{1} j_{2}}^{\text {cut }} \in(0.64,2.2) \mathrm{TeV}$ and $\Delta y_{j_{1} j_{2}}^{\text {cut }}<4$.8. We note that for arbitrary luminosities $L$, the significance can be calculated as $S(L)=S\left(L=1 \mathrm{fb}^{-1}\right) \cdot \sqrt{L \mathrm{fb}}$. 

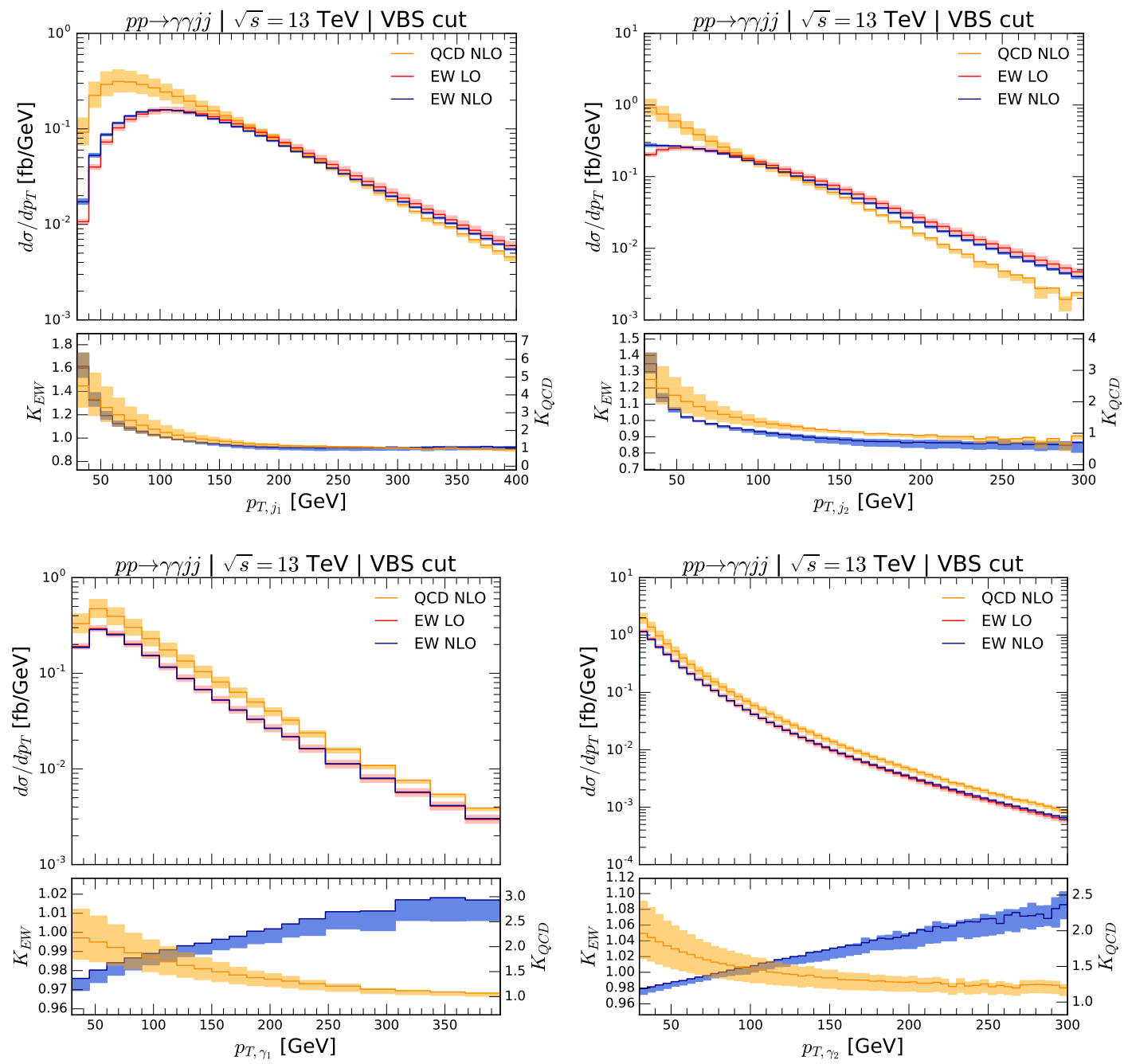

Figure 5. Distributions of the transverse momentum of the hardest jet (top left), the secondhardest jet (top right), the hardest photon (bottom left) and the second-hardest photon (bottom right). The scale-uncertainty bands are calculated from the maximum and minimum of $\left[d \sigma\left(H_{T} / 4\right), d \sigma\left(H_{T} / 2\right), d \sigma\left(H_{T}\right)\right]$ with $\mu_{R}=\mu_{F}$. In the small panels the $K$-factor defined as NLO/LO is shown.

It is also interesting to compare the $\gamma \gamma j j$ process with similar ones of $\ell^{+} \ell^{-} \gamma j j$ and $\ell \nu_{\ell} \gamma j j$ with $\ell=e, \mu$. This comparison is shown in table 4 . The default kinematic cuts used for the $\gamma \gamma j j$ process are also applied to the other ones. Additionally, to select the charged leptons we use

$$
\begin{aligned}
p_{T, \ell} & >30 \mathrm{GeV}, & \left|y_{\ell}\right| & <2.5, \\
\Delta R_{j, \ell} & >0.4, & \Delta R_{\ell \gamma} & >0.8 .
\end{aligned}
$$

We further require, for the $\ell^{+} \ell^{-} \gamma j j$ process,

$$
m_{\ell^{+} \ell^{-}}>15 \mathrm{GeV}, m_{\ell^{+} \ell^{-} \gamma}>120 \mathrm{GeV},
$$



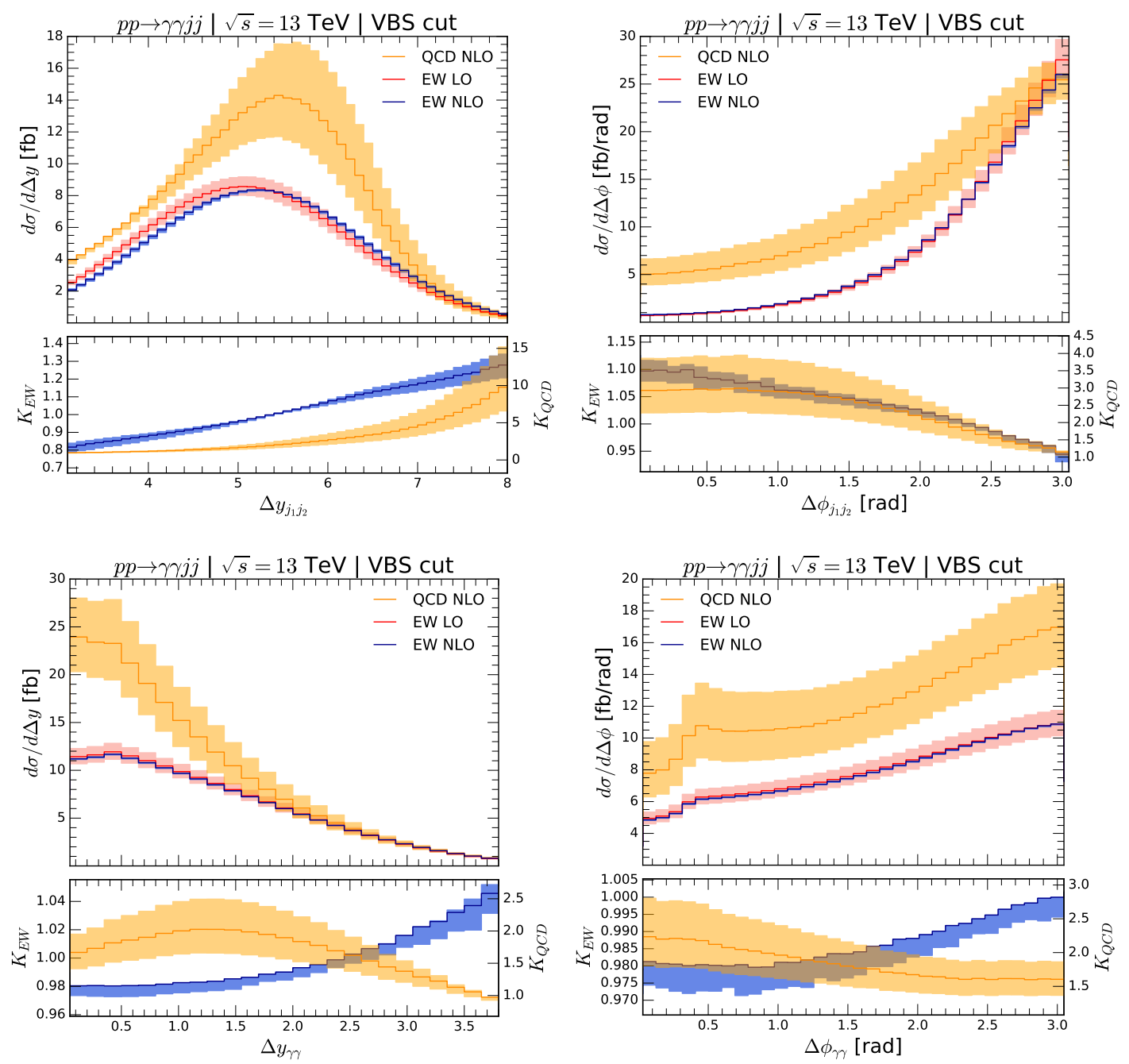

Figure 6. Distributions of the absolute value of the rapidity separation between the two tagging jets (top left) and between the two photons (bottom left), of the azimuthal-angle separation between the two tagging jets (top right) and between the two photons (bottom right). The scale-uncertainty bands and $K$-factors are calculated as in figure 5 .

to suppress the $\gamma^{\star} \rightarrow \ell^{+} \ell^{-}$and $Z \rightarrow \ell^{+} \ell^{-} \gamma$ contributions, respectively. The value of $120 \mathrm{GeV}$ was recommended in ref. [38]. For the $\ell \nu_{\ell} \gamma j j$ processes, following refs. [37, 66], we use

$$
m_{\ell \nu \gamma}^{T}=\sqrt{E_{T}^{2}-p_{T, \ell \nu \gamma}^{2}}>90 \mathrm{GeV}
$$

with $E_{T}=\sqrt{m_{\ell \gamma}^{2}+p_{T, \ell \gamma}^{2}}+p_{T, \nu}$ to suppress the $W \rightarrow \ell \nu_{\ell} \gamma$ contribution, which is, as the $Z \rightarrow \ell^{+} \ell^{-} \gamma$ one, a background to the anomalous-quartic-gauge-coupling measurements. Concerning the scale choice, similar to eq. (3.6) for the $\gamma \gamma j j$ case, we use $\mu_{F}=\mu_{R}=$ $H_{T}^{V \gamma} / 2$ with

$$
H_{T}^{V \gamma}=\sum_{i \in \text { partons }} p_{T, i}+p_{T, \gamma}+E_{T, V}
$$



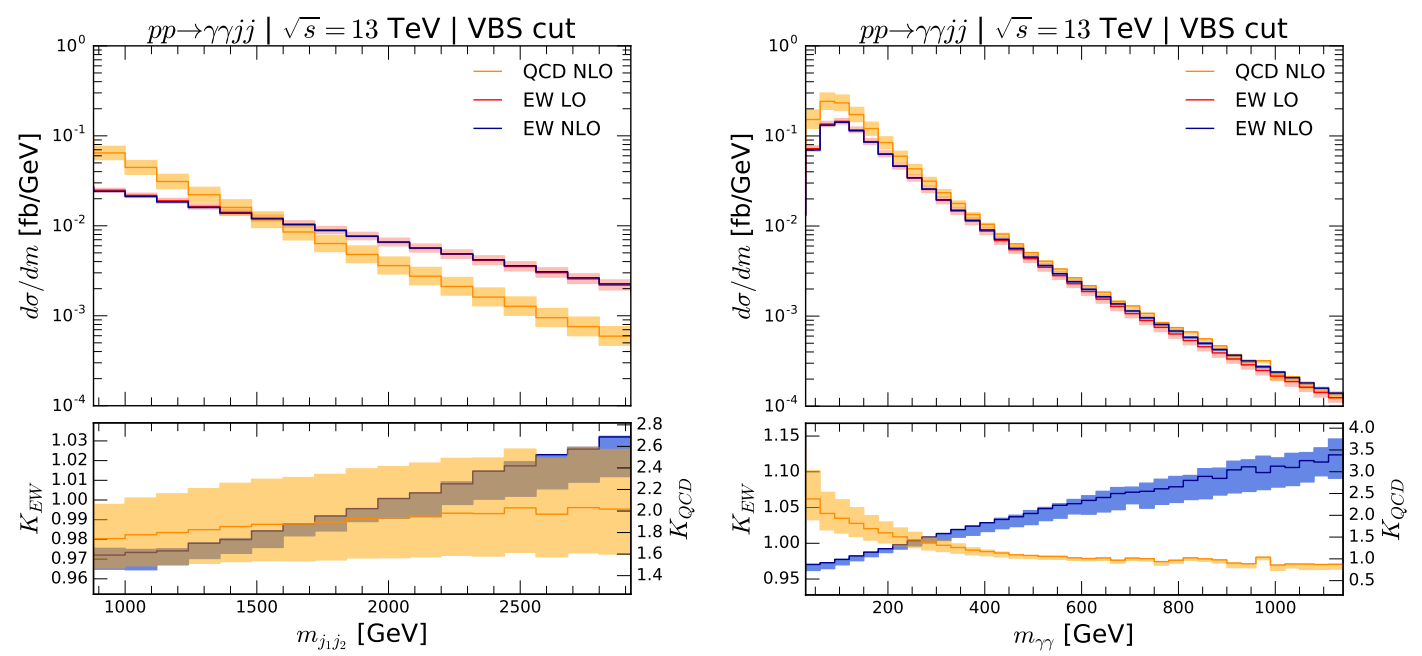

Figure 7. Distributions of the invariant mass of the two tagging jets (left) and of the two photons (right). The scale-uncertainty bands and $K$-factors are calculated as in figure 5 .

where $E_{T, V}=\sqrt{m_{V}^{2}+p_{T, V}^{2}}$ with $m_{V}$ being the reconstructed mass. With this setup, we have used VBFNLO version 3.0.0 beta 4, where the calculations of $\ell^{+} \ell^{-} \gamma j j \mathrm{EW}[36]$ and QCD [38] processes, as well as $\ell \nu_{\ell} \gamma j j \mathrm{EW}$ [35] and QCD [37] processes are included, to produce the NLO cross sections. We see that the significance for the $\gamma \gamma j j$ process is largest.

We now turn to differential cross sections. To understand the energy scale of the final state particles, we show in figure 5 the transverse momentum distributions of the tagging jets and the photons, individually, for the EW-induced channel at LO and NLO and for the QCD-induced process at NLO. Given the above cuts, the EW cross section is largest at $p_{T, j_{1}} \approx 110 \mathrm{GeV}, p_{T, j_{2}} \approx 40 \mathrm{GeV}, p_{T, \gamma_{1}} \approx 60 \mathrm{GeV}, p_{T, \gamma_{2}} \approx 35 \mathrm{GeV}$ at NLO. For the QCD background, the maximal position is at $p_{T, j_{1}} \approx 70 \mathrm{GeV}, p_{T, j_{2}} \approx 40 \mathrm{GeV}$, $p_{T, \gamma_{1}} \approx 60 \mathrm{GeV}, p_{T, \gamma_{2}} \approx 35 \mathrm{GeV}$. We observe that, for the photon distributions, the EW and QCD processes have the same shapes. However, for the jets, the QCD distribution falls faster than the EW one. On the small panels, the $K$-factors are shown for both EW and QCD processes. On all panels, the scale-uncertainty bands calculated from the maximum and minimum of $\left[d \sigma\left(H_{T} / 4\right), d \sigma\left(H_{T} / 2\right), d \sigma\left(H_{T}\right)\right]$ are plotted, where both scales are set equal. The $K$-factor bands are calculated from these maximum and minimum with a common normalization to the central LO cross section $d \sigma_{\mathrm{LO}}\left(H_{T} / 2\right)$. As expected, we see that, for the EW process, the NLO bands are much shrunk compared to the LO ones. We also see that the scale uncertainties on the QCD process are significantly larger than on the EW one. The $K$-factors of the QCD channel are also much larger. In the low $p_{T}$ region, the $K$-factors reach very large values, e.g. at $p_{T, j_{1}} \approx 40 \mathrm{GeV}$ we get $K_{\mathrm{QCD}} \approx 3.8, K_{\mathrm{EW}} \approx 1.3$. In the maximal cross section region, where $p_{T, j_{1}} \approx 100 \mathrm{GeV}$, those values change to 1.9 and 1.0, respectively. The rise of the $K$-factor in the small $p_{T}$ region comes from the twojet-exclusive real-radiation contribution for both EW and QCD processes, and it indicates that a fixed-order NLO calculation is not sufficient for reliable predictions in this region. This behavior has also been observed in other processes such as $W^{+} W^{+} j j[12,27]$ and 

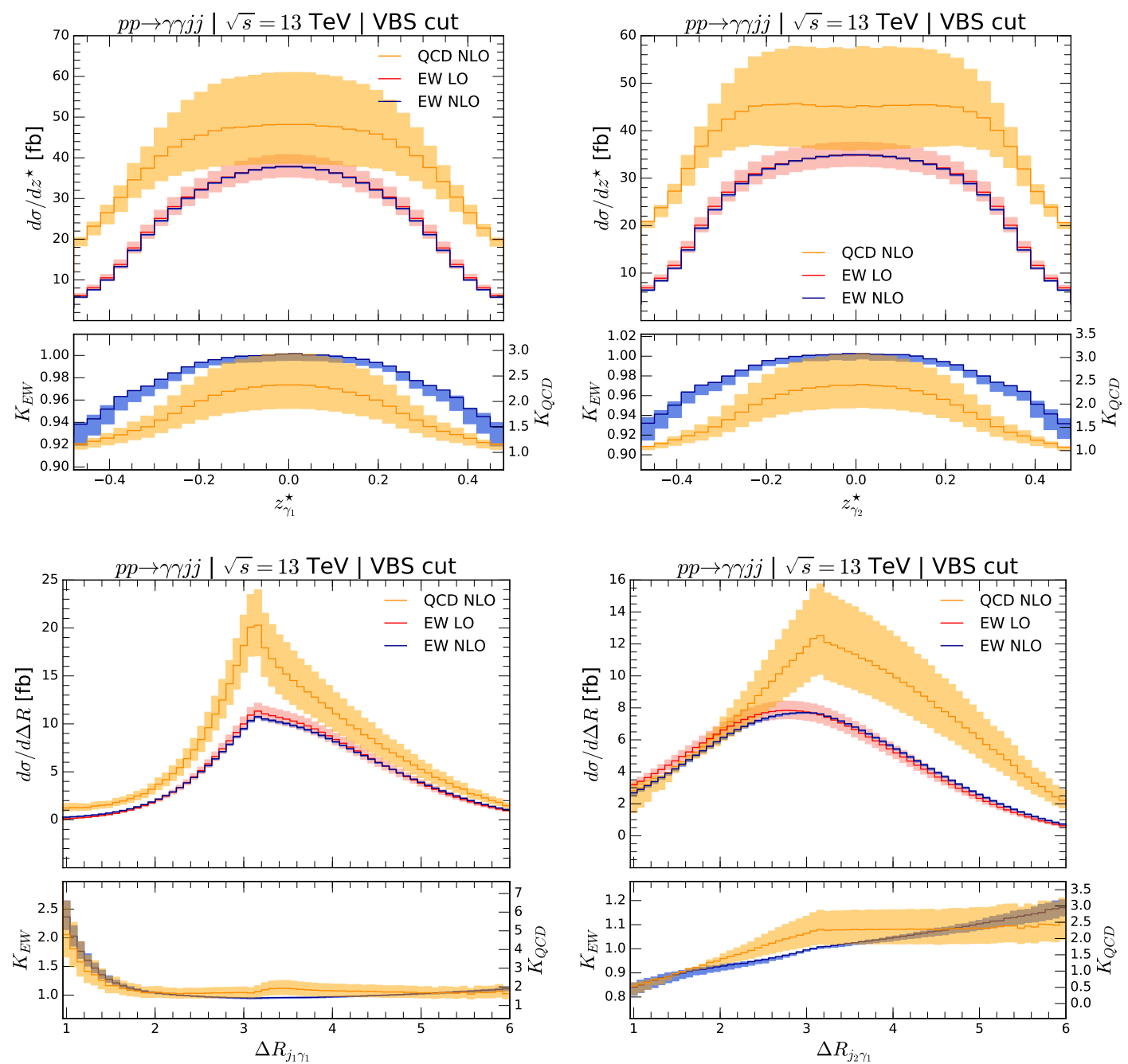

Figure 8. Distributions of the $z_{\gamma_{i}}^{\star}$ with $i=1,2$ (top row) defined in eq. (3.11) and of the $R$ separation between the hardest photon and the tagging jets (bottom row). The scale-uncertainty bands and $K$-factors are calculated as in figure 5 .

$Z_{\gamma j j}$ [38] (see ref. [12] for a detailed discussion). Since the calculation of the next-tonext-to-leading-order (NNLO) prediction of this process is beyond the current reach of higher order calculations, we propose the merging of NLO predictions with various jet multiplicities within a parton-shower framework to improve the accuracy of the prediction of the QCD channel, which we leave for a future work. For the EW signal, an NLO prediction is sufficient.

In order to see how the events look like, we show in figure 6 the rapidity and azimuthalangle separation between the two tagging jets and between the photons. The $\Delta y_{j_{1} j_{2}}$ plot is surprising. Normally, we expect that the most likely rapidity separation for the EW process is larger than for the QCD one, as shown in ref. [27] for the case of $W^{+} W^{+} j j$ production and in ref. [36] for $Z \gamma j j$. However, the plot in figure 6 (top left) shows the contrary: the QCD cross section peaks at $\Delta y_{j_{1} j_{2}} \approx 5.5$ while the EW one at about 5.3. This means that 

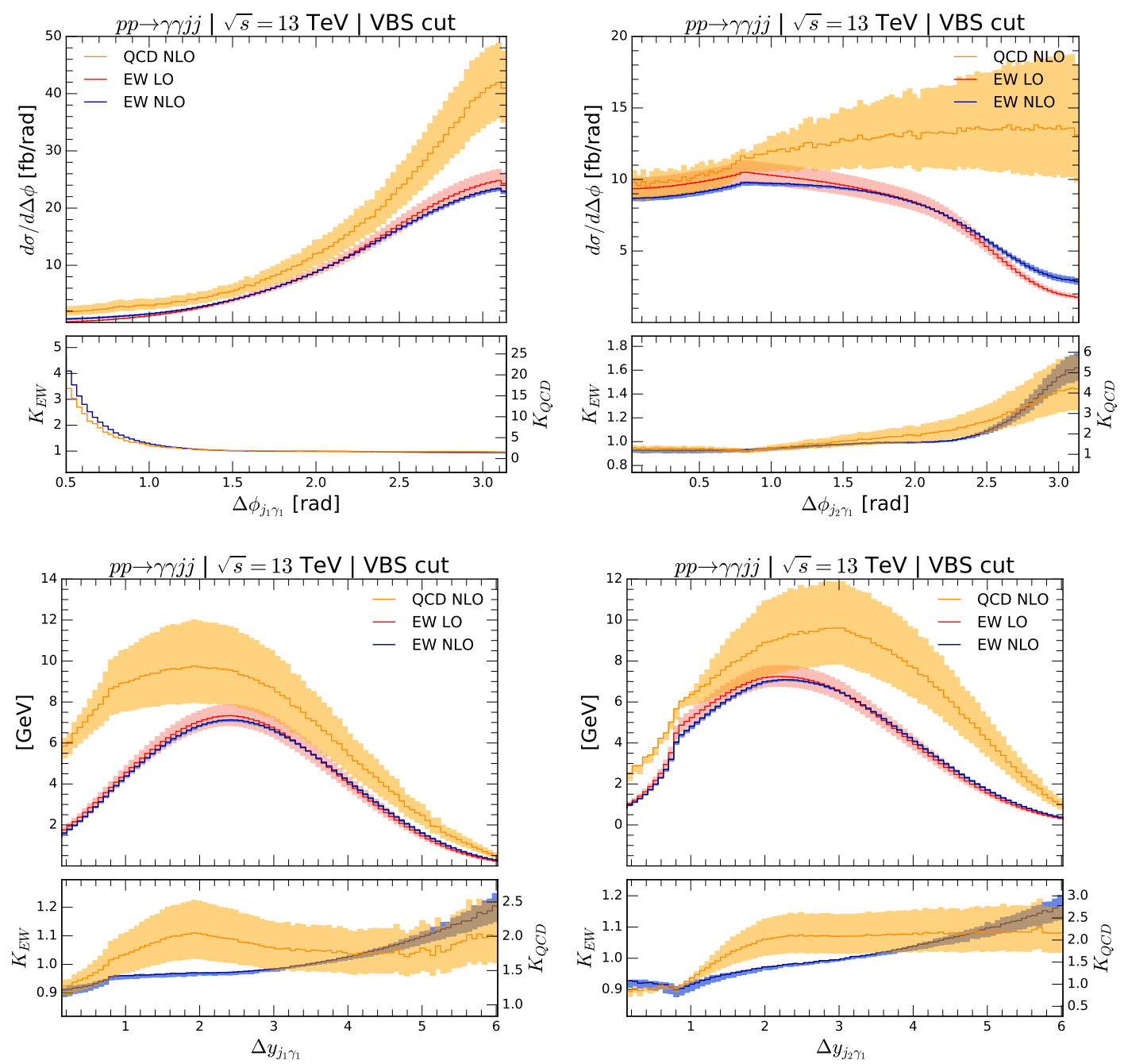

Figure 9. Distributions of the $\phi$-separation (top row) and the absolute value of the $y$-separation (bottom row) between the hardest photon and the tagging jets. The scale-uncertainty bands and $K$-factors are the same as figure 5 .

a large $\Delta y_{j_{1} j_{2}}$ cut is not efficient to enhance the signal-over-background ratio in this case. We have chosen the cut $\Delta y_{j_{1} j_{2}}>3$ as a default setting in this paper. However, the $\Delta y_{j_{1} j_{2}}$ distributions show that using a looser cut can be good as well. The difference between the QCD and EW channels is also very pronounced in the $\Delta \phi_{j_{1} j_{2}}$ distributions. Although both processes peak at $\Delta \phi_{j_{1} j_{2}} \approx \pi$ as expected, because the hardest jet is recoiling against other particles, the QCD cross section is more uniformly distributed than the EW one. Similar distributions for the two photons are also presented. The $\Delta y_{\gamma \gamma}$ plot shows that a small rapidity separation is the preferred configuration for both EW and QCD processes. The EW distribution has one local maximum at $\Delta y \approx 0.45$ then drops gently as the separation increases. The QCD distribution, being largest in the region $\Delta y \lesssim 0.45$, drops more rapidly than the EW one as the separation increases. The $\Delta \phi_{\gamma \gamma}$ distributions have a jump at $\Delta \phi \approx 0.4$ because of the $\Delta R_{\gamma \gamma}>0.4$ cut. The results show that a large $\Delta \phi$ separation 
is slightly preferred for both channels. Compared to the two-jet case, it is more uniformly distributed.

The distribution of the invariant mass of the two tagging jets and of the two photons are shown in figure 7. As expected, we see that the QCD-induced cross section drops more rapidly with increasing di-jet invariant mass than the EW-induced one. This justifies the high value of the $m_{j_{1} j_{2}}$ cut used in this paper. The EW $K$-factor is close to unity for a large range of the invariant mass, up to $3 \mathrm{TeV}$, suggesting that this distribution is perturbatively well behaved even at very high energies for the signal. For the QCD background, the $K$ factor is also rather constant, but much larger, slowly increasing from 1.7 to 2.0. This large $K$-factor together with a large uncertainty band again signal the importance of predictions beyond the fixed-order NLO accuracy for the QCD process. The $m_{\gamma \gamma}$ plot on the right shows that the EW NLO distribution peaks at $120 \mathrm{GeV}$ while the QCD at $90 \mathrm{GeV}$. We note that the Higgs contribution is not included in the EW channel as it is beyond the fixed-order corrections considered here. Concerning the $K$-factor, it is very close to unity for the EW process. For the QCD channel, it is large at small invariant masses, decreasing from 2.7 at $m_{\gamma \gamma} \approx 30 \mathrm{GeV}$, reaching 1.0 at around $600 \mathrm{GeV}$ then being rather constant after that.

To understand the jet-photon separations, we show in figure 8 the distributions of the $z_{\gamma_{i}}^{\star}$ with $i=1,2$ (top row) defined as

$$
z_{X}^{\star}=\frac{y_{X}-\left(y_{j_{1}}+y_{j_{2}}\right) / 2}{\left|y_{j_{1}}-y_{j_{2}}\right|}, \quad X \in\left(\gamma_{1}, \gamma_{2}, j_{3}\right),
$$

and of the $R$-separation between the hardest photon and the tagging jets (bottom row). The $z_{\gamma_{i}}^{\star}$ distributions show the distance of the photon with respect to the tagging jets with values of $1 / 2$ and $-1 / 2$ when the photon equals the rapidity of jet 1 and 2 , respectively. Due to the cuts imposed, we observe as expected that in both EW and QCD induced channels the photons are nearly homogeneously distributed in the center between the tagging jets. This has to be compared with the differential distribution of $z_{j_{3}}^{\star}$, computed here only at LO and not shown, where the third jet aligns in the EW-induced channel with either of the two leading jets while in the QCD-induced process, the distributions have a pronounced peak at $z_{j_{3}}^{\star}=0$. Note that, the two $z_{\gamma_{i}}^{\star}$ plots in figure 8 are not identical because the photons are ordered by $p_{T}$. As expected, the distributions are flatter for the softer photon.

The peaks in the $\Delta R_{j_{1} \gamma_{1}}$ distributions show that the preferred configuration is when the hardest jet and the hardest photon are back-to-back (i.e. $\Delta \phi_{j_{1} \gamma_{1}}=\pi$ ) for both channels. For small separations, the $K$-factors are large with $K \approx 2.2$ (4.8) for EW (QCD) induced mechanisms at $\Delta R \approx 1$. The $K$-factors then decrease steadily before reaching a constant value of $K \approx 1(2)$ for $\Delta R \geq 1.4$. The large values of the $K$-factors at small separations can be understood as follows. For this configuration to exist, the $j_{1}-\gamma_{1}$ system is mostly recoiling against the $j_{2}-\gamma_{2}-p_{3}$ system, where the parton $p_{3}$ can be a third jet or an unresolved parton (e.g. lost in the beams or having rapidity $y>5$ ). Thus, the large $K$-factors are due to this real-emission contribution and almost-vanishing LO cross sections. Note that, the threeparton contribution is only calculated at LO. This also explains why the scale-uncertainty bands are large at small separations. The $\Delta R_{j_{2} \gamma_{1}}$ distributions in the bottom-right plot 


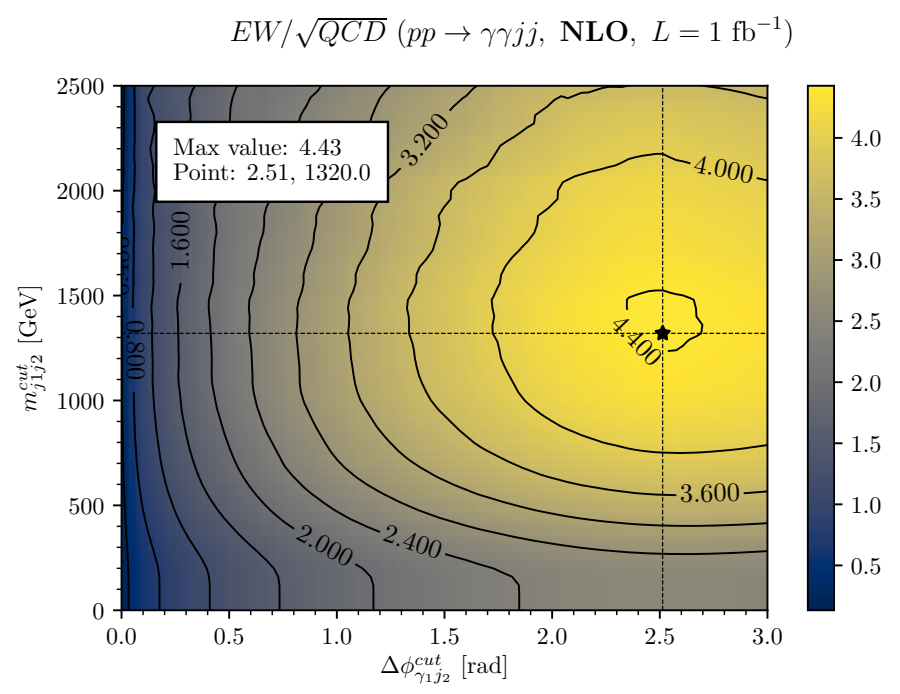

Figure 10. Dependence of the NLO significance EW $/ \sqrt{\mathrm{QCD}}$ calculated with luminosity $L=1 \mathrm{fb}^{-1}$ on the cuts $m_{j_{1} j_{2}}>m_{j_{1} j_{2}}^{\text {cut }}$ and $\Delta \phi_{j_{2} \gamma_{1}}<\Delta \phi_{j_{2} \gamma_{1}}^{\text {cut }}$. In addition, the other default cuts are used.

show that the events are more uniformly distributed compared to the $\Delta R_{j_{1} \gamma_{1}}$ distributions and this happens already at LO. As a consequence, the $K$-factors are more moderate. For the EW-induced channel, the $K$-factor increases steadily from 0.9 to 1.2 for $\Delta R \in(1,6)$. For the QCD case, the $K$-factor increases more rapidly from 0.5 to 2.0 for $\Delta R \in(1,3)$, then suddenly changes its behavior to be rather constant varying from 2.0 to 2.5 for $\Delta R \in(3,6)$. This sudden change at $\Delta R \approx 3$ is also visible in the $\Delta R_{j_{1} \gamma_{1}}$ case, but to a much lesser extent.

These findings are confirmed in figure 9 where the $\Delta \phi_{j_{i} \gamma_{1}}$-separation (top row) and $\Delta y_{j_{i} \gamma_{1}}$-separation (bottom row) of the hardest photon and tagging jets are shown. One can observe that the region of $\Delta R>3$ is mostly explained by $\Delta y$ while the $\Delta R<3$ region is an interplay of the two contributing observables $\Delta y$ and $\Delta \phi$. It is clearly visible in the upper plots that the real-emission contribution is dominant at around $\Delta \phi_{j_{1} \gamma_{1}}=0$ and $\Delta \phi_{j_{2} \gamma_{1}}=\pi$. For the QCD (EW)-induced mechanism, the $K$-factor is 16(4) at $\Delta \phi_{j_{1} \gamma_{1}}=0.5$, reaching values larger than 50(24) for $\Delta \phi_{j_{1} \gamma_{1}}<0.25$. In the upper right plot, we observe in the $\Delta \phi_{j_{2} \gamma_{1}}$ distribution that the $K$-factors are more moderate with values up to 4.3 (1.6) at $\pi$, however, the relative relevance is higher since the maximum $K$-factor is reached for the QCD-induced sample in the dominant region of the differential distribution. This region is dominated by three-parton events, computed only at LO, explaining the larger scale uncertainties. These large $K$-factors highlight the relevance of further radiation which can be studied at parton level at NNLO or including parton-shower effects and merging different jet multiplicities at NLO. We leave for future work the study of parton-shower effects in the framework of VBFNLO and Herwig. Additionally, this observable discriminates the QCDand EW-induced processes in the region of $\Delta \phi_{j_{2} \gamma_{1}}>2$. To study the discriminant capacities of the observable, in figure 10 , we show the NLO significance EW $/ \sqrt{\text { QCD }}$ calculated with luminosity $L=1 \mathrm{fb}^{-1}$ on the cuts $m_{j_{1} j_{2}}>m_{j_{1} j_{2}}^{\text {cut }}$ and $\Delta \phi_{j_{2} \gamma_{1}}<\Delta \phi_{j_{2} \gamma_{1}}^{\text {cut }}$. On top of this, the other default cuts are used. We observe a maximum of 4.43 at around $\Delta \phi_{j_{2} \gamma_{1}} \approx 2.51$ 


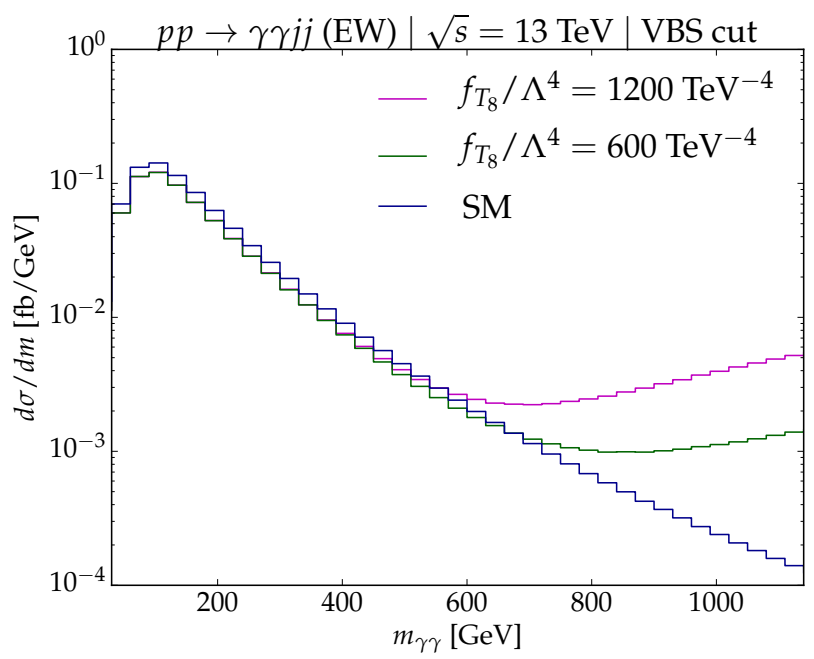

Figure 11. NLO distributions of the invariant mass of the two photons in the presence of anomalous gauge couplings with the dimension-8 operator defined in eq. (3.13).

and $m_{j_{1} j_{2}} \approx 1320 \mathrm{GeV}$, which has to be compared with the maximal value of 4.31 without the $\Delta \phi_{j_{2} \gamma_{1}}$ cut in figure 4 . For our default cuts, $m_{j_{1} j_{2}}>800 \mathrm{GeV}$ and $\Delta y_{j_{1} j_{2}}>3$, the significance changes from 4.0 to 4.1, if the additional cut is applied. While the significance does not increase considerably, we consider that this cut should be applied in the search for new physics since the region of the phase space removed is mainly dominated in the QCDinduced mechanism by three or more parton events as previously discussed, and, thus, more sensitive to higher order corrections. We note that after applying a cut of $\Delta \phi_{j_{2} \gamma_{1}}<2$ the integrated QCD $K$-factor decreases from 1.78 to 1.43 and at the differential level from 4.21 at $\pi$ to 1.93 at $\Delta \phi_{j_{2} \gamma_{1}}=2$. This effect is much less pronounced in the EW-induced mechanism with the changes of $K$-factor from 0.99 to 0.95 and from 1.66 to 0.99 at the integrated and differential cross-section level, respectively.

Finally, we show in figure 11 the $m_{\gamma \gamma}$ distribution in the presence of anomalous gauge couplings. As explained in refs. [18, 49], dimension- 6 and 8 operators are included for the set of EW-induced $p p \rightarrow V V^{\prime} j j$ processes in the VBFNLO program using the effective Lagrangian approach. This Lagrangian reads

$$
\mathcal{L}_{\mathrm{EFT}}=\mathcal{L}_{\mathrm{SM}}+\sum_{d=6,8} \sum_{i} \frac{f_{i}}{\Lambda^{d-4}} \mathcal{O}_{i}^{(d)}
$$

where the operators have been defined in refs. [49, 55, 67, 68]. For the illustration in figure 11 we turn on only the following operator

$$
\mathcal{L}_{\mathrm{EFT}}^{T_{8}}=\frac{f_{T_{8}}}{\Lambda^{4}} \hat{B}_{\mu \nu} \hat{B}^{\mu \nu} \hat{B}_{\alpha \beta} \hat{B}^{\alpha \beta},
$$

where $\hat{B}_{\mu \nu}=i g^{\prime}\left(\partial_{\mu} B_{\nu}-\partial_{\nu} B_{\mu}\right) / 2$ with $g^{\prime}$ and $B_{\mu}$ being the coupling and the gauge field associated with the $\mathrm{U}(1)_{Y}$ group, as defined in ref. [49]. We note that other operators defined in refs. $[18,49]$ are included as usual in the EW-induced $\gamma \gamma j j$ process. However, not all dimension- 6 and 8 operators are included. For example, operators with fermionic 
fields are not taken into account. We have selected the operator in eq. (3.13) because it induces tree-level $\gamma \gamma \gamma \gamma, \gamma \gamma \gamma Z, \gamma \gamma Z Z$ couplings which are absent in the SM. As default in VBFNLO, the anomalous terms of order $\mathcal{O}\left(1 / \Lambda^{8}\right)$ are kept. This guarantees that the LO cross section is always positive. At NLO, corrections of order $\mathcal{O}\left(\alpha_{s} / \Lambda^{8}\right)$ are consistently included. With the values of $f_{T_{8}} / \Lambda^{4}=600$ and $1200 \mathrm{TeV}^{-4}$ satisfying the current experimental bound [31] (without introducing form factors), we see in figure 11 that the di-photon invariant mass distribution is very sensitive to this operator at high energies.

\section{Conclusions}

Results at NLO QCD for photon pair production in association with two jets via vector boson scattering have been presented for the first time in this paper. We also showed results for the QCD-induced mechanism finding good agreement with the previous calculations. In order to guarantee the validity of the VBS approximation we remove the $s$-channel contributions using the cut of eq. (3.5) on top of a tight VBS-cut setup. With these cuts, the VBS approximation is almost identical to the full EW result and the EW-QCD interference is negligible. We have investigated the dependence of the cross sections on the photon-isolation parameters. Our results show that choosing the cone radius $\delta_{0}=0.4$ and $\epsilon=0.05$ as suggested in ref. [59] in the context of inclusive diphoton production is also good for the $\gamma \gamma j j$ channel.

To increase the signal versus background ratio $S=\mathrm{EW} / \sqrt{\mathrm{QCD}}$, we studied the dependence of the cross sections for the two production mechanisms on the two typical VBS cuts $m_{j_{1} j_{2}}$ and $\Delta y_{j_{1} j_{2}}$. We find that tight VBS cuts, in particular a large $m_{j_{1} j_{2}}$ cut, are needed in order to optimize the significance, which turns out to be higher than for other VBS processes. We used as a default setup $m_{j_{1} j_{2}}>800 \mathrm{GeV},\left|y_{j_{1}}-y_{j_{2}}\right|>3$ and the two photons are required to be between the rapidity gap of the two tagging jets to obtain a significance of about 4. A higher significance of 4.3 is found for $m_{j_{1} j_{2}}>1300 \mathrm{GeV}$ and $\Delta y_{j_{1} j_{2}}>3$ with an integrated cross section of $\sigma_{\mathrm{NLO}}^{\mathrm{EW}}=13.35 \mathrm{fb}$. A big plateau with $S>4.25$ is identified for $m_{j_{1} j_{2}}^{\text {cut }} \in(1,1.6) \mathrm{TeV}$ and $\Delta y_{j_{1} j_{2}}^{\text {cut }}<3.5$. Furthermore, we observe that applying a cut of $\Delta \phi_{j_{2} \gamma_{1}}^{\text {cut }}<2.5$ not only increases the significance a little bit to 4.43 , but, as well, reduces drastically the impact of higher-order QCD corrections.

For the EW-induced process, using the default cuts, different scale choices have been studied and we find that the scale dependence is significantly smaller for all choices when the NLO corrections are included - from about $15 \%$ at LO to a few percent or less at NLO QCD when varying both scales simultaneously $\mu_{F}=\mu_{R}$ by a factor of 2 around the central scale. Additionally, at the central scale, we observe that the integrated NLO QCD predictions for different scale choices are as well consistent among each other at the percent level, while at LO larger differences up to $10 \%$ level are visible. This highlights the relevance of the NLO QCD predictions. We find that the scales $H_{T} / 2$ and $\sqrt{p_{T, j_{1}} p_{T, j_{2}}}$ provide the most stable results with $K$-factors close to 1 at the integrated cross section level. With our default scale, $H_{T} / 2$, we studied the effect of the NLO QCD corrections at the differential cross section level. With our VBS default cuts, for the VBS channel, corrections are small, at the a-few-percent level, for EW observables in the whole spectrum while larger corrections up to $50 \%$ are visible for jet observables. These large corrections occur in the 
region of phase space where the LO cross section is suppressed due to kinematic reasons or when the $p_{T}$ of the tagging jets are small. For the QCD-induced mechanism, corrections can be much larger reaching $K$-factor of 10 or higher (see the $\Delta \phi_{j_{1} \gamma_{1}}$ distribution).

In addition, we have included in our code dimension- 6 and 8 operators involving EW gauge bosons and the Higgs field using an effective Lagrangian framework. We have shown, as an illustrative example, in figure 11 the capability of our program to study anomalousquartic-gauge couplings at NLO QCD. The code will be available in the next release of the VBFNLO program or upon request.

With the results obtained, we see that one of the main challenges to achieve a precise EW $\gamma \gamma j j$ measurement is to reduce the theoretical uncertainties of the QCD process. Since the NNLO corrections for this $2 \rightarrow 4$ process is unlikely to be available in the near future, further studies including parton-shower effects or using the all-order resummation discussed in ref. [69] for the case of $H j j$ can be valuable.

\section{Acknowledgments}

FC and IR acknowledge financial support by the Generalitat Valenciana, Spanish Government and ERDF funds from the European Commission (Grants No. RYC-201416061, SEJI-2017/2017/019, FPA2017-84543-P, FPA2017-84445-P, and SEV-2014-0398). The research of MK is supported by the Swiss National Science Foundation (SNF) under grant number 200020-175595. The work of LDN is funded by the Vietnam National Foundation for Science and Technology Development (NAFOSTED) under grant number 103.01-2017.78.

Open Access. This article is distributed under the terms of the Creative Commons Attribution License (CC-BY 4.0), which permits any use, distribution and reproduction in any medium, provided the original author(s) and source are credited.

\section{References}

[1] T. Figy, C. Oleari and D. Zeppenfeld, Next-to-leading order jet distributions for Higgs boson production via weak boson fusion, Phys. Rev. D 68 (2003) 073005 [hep-ph/0306109] [INSPIRE].

[2] ATLAS collaboration, Observation of electroweak production of a same-sign $W$ boson pair in association with two jets in pp collisions at $\sqrt{s}=13 \mathrm{TeV}$ with the ATLAS detector, Phys. Rev. Lett. 123 (2019) 161801 [arXiv:1906.03203] [INSPIRE].

[3] CMS collaboration, Observation of electroweak production of same-sign $W$ boson pairs in the two jet and two same-sign lepton final state in proton-proton collisions at $\sqrt{s}=13 \mathrm{TeV}$, Phys. Rev. Lett. 120 (2018) 081801 [arXiv: 1709. 05822] [INSPIRE].

[4] ATLAS collaboration, Observation of electroweak $W^{ \pm} Z$ boson pair production in association with two jets in pp collisions at $\sqrt{s}=13$ TeV with the ATLAS detector, Phys. Lett. B 793 (2019) 469 [arXiv: 1812.09740] [INSPIRE].

[5] CMS collaboration, Measurement of electroweak $W Z$ boson production and search for new physics in $W Z+$ two jets events in pp collisions at $\sqrt{s}=13$ TeV, Phys. Lett. B 795 (2019) 281 [arXiv: 1901.04060] [INSPIRE]. 
[6] CMS collaboration, Measurement of vector boson scattering and constraints on anomalous quartic couplings from events with four leptons and two jets in proton-proton collisions at $\sqrt{s}=13$ TeV, Phys. Lett. B 774 (2017) 682 [arXiv:1708.02812] [INSPIRE].

[7] B. Jager, C. Oleari and D. Zeppenfeld, Next-to-leading order QCD corrections to $W^{+} W^{-}$ production via vector-boson fusion, JHEP 07 (2006) 015 [hep-ph/0603177] [INSPIRE].

[8] B. Jager, C. Oleari and D. Zeppenfeld, Next-to-leading order QCD corrections to Z boson pair production via vector-boson fusion, Phys. Rev. D 73 (2006) 113006 [hep-ph/0604200] [INSPIRE].

[9] G. Bozzi, B. Jager, C. Oleari and D. Zeppenfeld, Next-to-leading order QCD corrections to $W^{+} Z$ and $W^{-} Z$ production via vector-boson fusion, Phys. Rev. D 75 (2007) 073004 [hep-ph/0701105] [INSPIRE].

[10] B. Jager, C. Oleari and D. Zeppenfeld, Next-to-leading order QCD corrections to $W^{+} W^{+} j j$ and $W^{-} W^{-} j j$ production via weak-boson fusion, Phys. Rev. D 80 (2009) 034022 [arXiv: 0907.0580] [INSPIRE].

[11] A. Denner, L. Hosekova and S. Kallweit, NLO QCD corrections to $W^{+} W^{+} j$ j production in vector-boson fusion at the LHC, Phys. Rev. D 86 (2012) 114014 [arXiv:1209.2389] [INSPIRE].

[12] B. Biedermann, A. Denner and M. Pellen, Complete NLO corrections to $W^{+} W^{+}$scattering and its irreducible background at the LHC, JHEP 10 (2017) 124 [arXiv:1708.00268] [INSPIRE].

[13] A. Ballestrero et al., Precise predictions for same-sign W-boson scattering at the LHC, Eur. Phys. J. C 78 (2018) 671 [arXiv:1803.07943] [InSPIRE].

[14] A. Denner et al., QCD and electroweak corrections to WZ scattering at the LHC, JHEP 06 (2019) 067 [arXiv: 1904.00882] [INSPIRE].

[15] B. Feigl, Electroweak processes in the standard model and beyond: backgrounds to Higgs physics and semileptonic decay modes, Ph.D. thesis, KIT, Karlsruhe, Germany (2013).

[16] K. Arnold et al., VBFNLO: a parton level Monte Carlo for processes with electroweak bosons, Comput. Phys. Commun. 180 (2009) 1661 [arXiv:0811.4559] [INSPIRE].

[17] J. Baglio et al., VBFNLO: a parton level Monte Carlo for processes with electroweak bosons - Manual for Version 2.7.0, arXiv:1107.4038 [INSPIRE].

[18] J. Baglio et al., Release Note - VBFNLO 2.7.0, arXiv:1404.3940 [InSPIRE].

[19] V. Hankele and D. Zeppenfeld, QCD corrections to hadronic WWZ production with leptonic decays, Phys. Lett. B 661 (2008) 103 [arXiv:0712.3544] [InSPIRE].

[20] F. Campanario et al., QCD corrections to charged triple vector boson production with leptonic decay, Phys. Rev. D 78 (2008) 094012 [arXiv:0809.0790] [INSPIRE].

[21] G. Bozzi, F. Campanario, V. Hankele and D. Zeppenfeld, NLO QCD corrections to $W^{+} W^{-} \gamma$ and $Z Z \gamma$ production with leptonic decays, Phys. Rev. D 81 (2010) 094030 [arXiv:0911.0438] [INSPIRE].

[22] G. Bozzi, F. Campanario, M. Rauch, H. Rzehak and D. Zeppenfeld, NLO QCD corrections to $W^{ \pm} Z \gamma$ production with leptonic decays, Phys. Lett. B 696 (2011) 380 [arXiv:1011.2206] [INSPIRE].

[23] T. Melia, K. Melnikov, R. Rontsch and G. Zanderighi, Next-to-leading order QCD predictions for $W^{+} W^{+} j j$ production at the LHC, JHEP 12 (2010) 053 [arXiv:1007.5313] [INSPIRE]. 
[24] T. Melia, K. Melnikov, R. Rontsch and G. Zanderighi, $N L O$ QCD corrections for $W^{+} W^{-}$ pair production in association with two jets at hadron colliders, Phys. Rev. D 83 (2011) 114043 [arXiv: 1104.2327] [INSPIRE].

[25] N. Greiner et al., NLO QCD corrections to the production of $W^{+} W^{-}$plus two jets at the LHC, Phys. Lett. B 713 (2012) 277 [arXiv:1202.6004] [InSPIRE].

[26] F. Campanario, M. Kerner, L.D. Ninh and D. Zeppenfeld, $W Z$ production in association with two jets at next-to-leading order in QCD, Phys. Rev. Lett. 111 (2013) 052003 [arXiv: 1305.1623] [INSPIRE].

[27] F. Campanario, M. Kerner, L.D. Ninh and D. Zeppenfeld, Next-to-leading order $Q C D$ corrections to $W^{+} W^{+}$and $W^{-} W^{-}$production in association with two jets, Phys. Rev. D 89 (2014) 054009 [arXiv: 1311.6738] [INSPIRE].

[28] F. Campanario, M. Kerner, L.D. Ninh and D. Zeppenfeld, Next-to-leading order QCD corrections to $Z Z$ production in association with two jets, JHEP 07 (2014) 148 [arXiv: 1405.3972] [INSPIRE].

[29] M. Chiesa, A. Denner, J.-N. Lang and M. Pellen, An event generator for same-sign $W$-boson scattering at the LHC including electroweak corrections, Eur. Phys. J. C 79 (2019) 788 [arXiv: 1906.01863] [INSPIRE].

[30] B. Biedermann, A. Denner and M. Pellen, Large electroweak corrections to vector-boson scattering at the Large Hadron Collider, Phys. Rev. Lett. 118 (2017) 261801 [arXiv: 1611.02951] [INSPIRE].

[31] ATLAS collaboration, Studies of $Z \gamma$ production in association with a high-mass dijet system in pp collisions at $\sqrt{s}=8 \mathrm{TeV}$ with the ATLAS detector, JHEP 07 (2017) 107 [arXiv: 1705. 01966] [INSPIRE].

[32] ATLAS collaboration, Evidence for electroweak production of two jets in association with a $Z \gamma$ pair in pp collisions at $\sqrt{s}=13 \mathrm{TeV}$ with the ATLAS detector, Phys. Lett. B 803 (2020) 135341 [arXiv: 1910.09503] [INSPIRE].

[33] CMS collaboration, Measurement of the cross section for electroweak production of $Z \gamma$ in association with two jets and constraints on anomalous quartic gauge couplings in proton-proton collisions at $\sqrt{s}=8 \mathrm{TeV}$, Phys. Lett. B 770 (2017) 380 [arXiv:1702.03025] [INSPIRE].

[34] CMS collaboration, Measurement of electroweak-induced production of $W \gamma$ with two jets in pp collisions at $\sqrt{s}=8 \mathrm{TeV}$ and constraints on anomalous quartic gauge couplings, JHEP 06 (2017) 106 [arXiv: 1612.09256] [INSPIRE].

[35] F. Campanario, N. Kaiser and D. Zeppenfeld, $W \gamma$ production in vector boson fusion at $N L O$ in QCD, Phys. Rev. D 89 (2014) 014009 [arXiv: 1309.7259] [INSPIRE].

[36] F. Campanario, M. Kerner and D. Zeppenfeld, $Z \gamma$ production in vector-boson scattering at next-to-leading order QCD, JHEP 01 (2018) 160 [arXiv:1704.01921] [INSPIRE].

[37] F. Campanario, M. Kerner, L.D. Ninh and D. Zeppenfeld, Next-to-leading order QCD corrections to $W \gamma$ production in association with two jets, Eur. Phys. J. C 74 (2014) 2882 [arXiv: 1402.0505] [INSPIRE].

[38] F. Campanario, M. Kerner, L.D. Ninh and D. Zeppenfeld, $Z \gamma$ production in association with two jets at next-to-leading order QCD, Eur. Phys. J. C 74 (2014) 3085 [arXiv:1407.7857] [INSPIRE]. 
[39] T. Gehrmann, N. Greiner and G. Heinrich, Precise QCD predictions for the production of a photon pair in association with two jets, Phys. Rev. Lett. 111 (2013) 222002 [arXiv:1308.3660] [INSPIRE].

[40] S. Badger, A. Guffanti and V. Yundin, Next-to-leading order QCD corrections to di-photon production in association with up to three jets at the Large Hadron Collider, JHEP 03 (2014) 122 [arXiv: 1312.5927] [inSPIRE].

[41] Z. Bern et al., Next-to-leading order $\gamma \gamma+2$-jet production at the LHC, Phys. Rev. D 90 (2014) 054004 [arXiv: 1402.4127] [InSPIRE].

[42] J. Bellm et al., HERWIG 7.0/HERWIG++ 3.0 release note, Eur. Phys. J. C 76 (2016) 196 [arXiv: 1512.01178] [INSPIRE].

[43] S. Frixione, Isolated photons in perturbative QCD, Phys. Lett. B 429 (1998) 369 [hep-ph/9801442] [INSPIRE].

[44] K. Hagiwara and D. Zeppenfeld, Amplitudes for multiparton processes involving a current at $e^{+} e^{-}, e^{ \pm} p$ and hadron colliders, Nucl. Phys. B 313 (1989) 560 [INSPIRE].

[45] F. Campanario, Towards $p p \rightarrow V V j j$ at $N L O$ QCD: bosonic contributions to triple vector boson production plus jet, JHEP 10 (2011) 070 [arXiv: 1105.0920] [INSPIRE].

[46] J. Alwall et al., MadGraph/MadEvent v4: the new web generation, JHEP 09 (2007) 028 [arXiv:0706.2334] [INSPIRE].

[47] T. Gleisberg et al., Event generation with SHERPA 1.1, JHEP 02 (2009) 007 [arXiv: 0811.4622] [INSPIRE].

[48] ShERPA collaboration, Event generation with Sherpa 2.2, SciPost Phys. 7 (2019) 034 [arXiv: 1905.09127] [INSPIRE].

[49] M. Rauch, Vector-boson fusion and vector-boson scattering, arXiv:1610.08420 [INSPIRE].

[50] C. Degrande et al., Monte Carlo tools for studies of non-standard electroweak gauge boson interactions in multi-boson processes: a snowmass white paper, arXiv:1309.7890 [INSPIRE].

[51] N.D. Christensen and C. Duhr, FeynRules - Feynman rules made easy, Comput. Phys. Commun. 180 (2009) 1614 [arXiv:0806.4194] [INSPIRE].

[52] N.D. Christensen et al., A Comprehensive approach to new physics simulations, Eur. Phys. J. C 71 (2011) 1541 [arXiv:0906.2474] [INSPIRE].

[53] http://feynrules.irmp.ucl.ac.be/wiki/EWdim6

[54] C. Degrande et al., Effective field theory: a modern approach to anomalous couplings, Annals Phys. 335 (2013) 21 [arXiv:1205.4231] [INSPIRE].

[55] O.J.P. Eboli, M.C. Gonzalez-Garcia and J.K. Mizukoshi, $p p \rightarrow j j e^{ \pm} \mu^{ \pm} \nu \nu$ and $j j e^{ \pm} \mu^{\mp} \nu \nu$ at $O\left(\alpha_{\mathrm{em}}^{6}\right)$ and $O\left(\alpha_{\mathrm{em}}^{4} \alpha_{s}^{2}\right)$ for the study of the quartic electroweak gauge boson vertex at CERN LHC, Phys. Rev. D 74 (2006) 073005 [hep-ph/0606118] [INSPIRE].

[56] http://feynrules.irmp.ucl.ac.be/wiki/AnomalousGaugeCoupling

[57] Particle Data Group collaboration, Review of particle physics, Phys. Rev. D 98 (2018) 030001 [INSPIRE].

[58] M. Cacciari, G.P. Salam and G. Soyez, The anti- $k_{t}$ jet clustering algorithm, JHEP 04 (2008) 063 [arXiv: 0802.1189] [INSPIRE].

[59] L. Cieri, Diphoton isolation studies, Nucl. Part. Phys. Proc. 273-275 (2016) 2033 [arXiv: 1510.06873] [INSPIRE]. 
[60] F. Campanario et al., Stress testing the vector-boson-fusion approximation in multijet final states, Phys. Rev. D 98 (2018) 033003 [arXiv: 1802.09955] [INSPIRE].

[61] A. Buckley et al., LHAPDF6: parton density access in the LHC precision era, Eur. Phys. J. C 75 (2015) 132 [arXiv: 1412.7420] [INSPIRE].

[62] J. Butterworth et al., PDF 4LHC recommendations for LHC Run II, J. Phys. G 43 (2016) 023001 [arXiv: 1510.03865] [INSPIRE].

[63] S. Dulat et al., New parton distribution functions from a global analysis of quantum chromodynamics, Phys. Rev. D 93 (2016) 033006 [arXiv: 1506.07443] [INSPIRE].

[64] L.A. Harland-Lang, A.D. Martin, P. Motylinski and R.S. Thorne, Parton distributions in the LHC era: MMHT 2014 PDFs, Eur. Phys. J. C 75 (2015) 204 [arXiv: 1412.3989] [InSPIRE].

[65] NNPDF collaboration, Parton distributions for the LHC Run II, JHEP 04 (2015) 040 [arXiv: 1410.8849] [INSPIRE].

[66] U. Baur, T. Han and J. Ohnemus, QCD corrections to hadronic $W \gamma$ production with nonstandard $W W \gamma$ couplings, Phys. Rev. D 48 (1993) 5140 [hep-ph/9305314] [INSPIRE].

[67] K. Hagiwara, R. Szalapski and D. Zeppenfeld, Anomalous Higgs boson production and decay, Phys. Lett. B 318 (1993) 155 [hep-ph/9308347] [INSPIRE].

[68] K. Hagiwara, S. Ishihara, R. Szalapski and D. Zeppenfeld, Low-energy effects of new interactions in the electroweak boson sector, Phys. Rev. D 48 (1993) 2182 [InSPIRE].

[69] J.R. Andersen et al., Higgs-boson plus dijets: higher-order matching for high-energy predictions, JHEP 08 (2018) 090 [arXiv: 1805.04446] [INSPIRE]. 